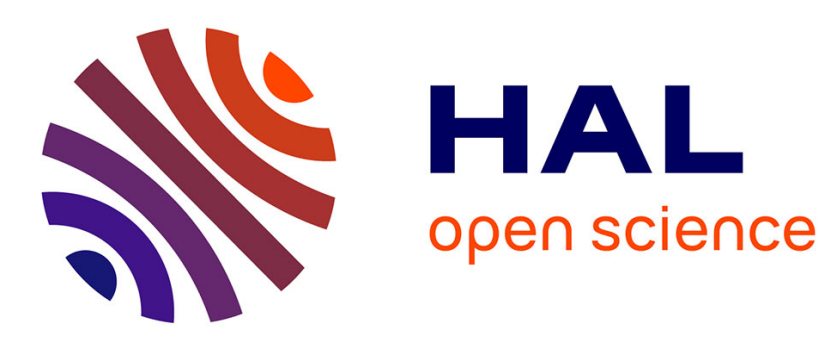

\title{
Observation de chloroplastes résistants aux triazines chez une panicoïdée, Setaria viridis $L$
} Jacques Gasquez, Jean-Pierre Compoint

\section{To cite this version:}

Jacques Gasquez, Jean-Pierre Compoint. Observation de chloroplastes résistants aux triazines chez une panicoïdée, Setaria viridis L. Agronomie, 1981, 1 (10), pp.923-926. hal-00884213

\section{HAL Id: hal-00884213 \\ https://hal.science/hal-00884213}

Submitted on 1 Jan 1981

HAL is a multi-disciplinary open access archive for the deposit and dissemination of scientific research documents, whether they are published or not. The documents may come from teaching and research institutions in France or abroad, or from public or private research centers.
L'archive ouverte pluridisciplinaire HAL, est destinée au dépôt et à la diffusion de documents scientifiques de niveau recherche, publiés ou non, émanant des établissements d'enseignement et de recherche français ou étrangers, des laboratoires publics ou privés. 


\section{Observation de chloroplastes résistants aux triazines chez une panicoïdée, Setaria viridis L.}

Jacques GASQUEZ \& Jean-Pierre COMPOINT

I.N.R.A., Laboratoire de Malherbologie, BV 1540, 21034 Dijon Cedex.

\section{RÉSUMÉ}

Mauvaise herbe, Setaria viridis $L$., Résistance chloroplastique, Résistance aux triazines, Détoxication des triazines.
Chez une espèce de la tribu des Panicoidées (Setaria viridis L.) qui métabolise plus ou moins l'atrazine, les auteurs ont trouvé une population qui n'avait pas été détruite par une très forte dose d'herbicide. Ces plantes particulières ne métabolisent pas plus d'atrazine que les individus d'une population jamais traitée mais possèdent des chloroplastes résistants : cette résistance est du même type que celle qui a été décrite chez des Dicotylédones. Les auteurs discutent les hypothèses traduisant l'évolution qui a pu conduire à la sélection de ce type d'individus malgré l'existence d'autres types de résistance.

\section{SUMMARY}

Weeds,

Setaria viridis $L$.,

Chloroplastic resistance, Triazines resistance, Triazines detoxification.
Evidence of triazine resistant chloroplasts within panicoideae : Setaria viridis $L$.

Within a species which can detoxify atrazine (Setaria viridis), the authors have found a population which was not controlled by a high level of triazine. These plants do not metabolize more atrazine than a never treated population but they have triazine resistant chloroplasts. So these plants have the same triazine resistance recently described within some susceptible species. The authors discuss the evolutionary process which gave rise to this mechanism of resistance among plants which were probably selected by another mechanism.

\section{INTRODUCTION}

Certaines Graminées, appartenant surtout à la tribu des Panicoïdées, grâce à la possibilité qu'elles ont de dégrader des quantités variables d'atrazine, résistent plus ou moins aux doses d'utilisation de ce produit (JENSEN et al., 1977). Cependant, les espèces du genre Setaria, qui métabolisent en moyenne toutes d'une manière comparable (THOMPSON, 1972), ne sont généralement capables de résister qu'à des doses un peu inférieures à celles qui sont habituellement utilisées pour les traitements des champs de maïs et sont incapables de survivre à des doses supérieures.

$\mathrm{Au}$ début de l'utilisation des triazines, la métabolisation de l'herbicide était la seule résistance connue (SHIMABU. KURO, 1968) et il est probable qu'il n'existait pas de populations présentant un autre type de résistance. La première observation d'un biotype résistant à des doses très élevées de triazines est celle de RYAN en $1970 \mathrm{chez}$ Senecio vulgaris. Dans les années qui ont suivi, des biotypes aussi résistants ont été signalés chez d'autres espèces et c'est en 1977 qu'il a été démontré que ces biotypes présentent une résistance liée à une grande insensibilité des chloroplastes dont le transfert photosystème II - photosystème I n'est pas bloqué (Souza MACHADo et al., 1977). De plus, toutes les populations observées résistent non seulement à l'atrazine, responsable de leur sélection, mais aussi à toutes les triazines (SoUZA MACHADO et al., 1978). Aujourd'hui, tant en Amérique qu'en Europe, ce type de résistance a été signalé chez une ou plusieurs populations d'une quinzaine d'espèces (AIGLE et al., 1980). Il est cependant remarquable de noter que ces espèces sont essentiellement des Dicotylédones. Une seule est une graminée, Poa annua (DUCRUET \& Gasquez, 1978). Ce qui rend le comportement de ces populations résistantes plus spectaculaire, c'est que toutes ces espèces incapables de dégrader les triazines sont normalement extrêmement sensibles.

Nous avons récolté une population de Setaria viridis dont les individus ont résisté à des doses triples de la normale (1500 g/ha). Un tel comportement nous a amenés à comparer l'absorption et la détoxification de l'atrazine chez ce biotype à celles d'une population sensible n'ayant jamais été traitée. En effet, tous les individus d'une même espèce n'ayant pas le même niveau de résistance, celle-ci pourrait être due à la sélection progressive d'individus plus résistants (GASQuez \& CompoinT, 1977). Par ailleurs, nous avons recherché si cette population pouvait présenter les caractéristiques de la résistance chloroplastique dont la manifestation au champ est la résistance à de fortes doses d'herbicide. 
Pour déterminer les caractéristiques de cette population, nous avons, d'une part, estimé le niveau de détoxication par la mesure de l'atrazine restant dans les plantes plusieurs jours après un traitement et, d'autre part, mesuré l'activité de chloroplastes isolés et observé la fluorescence de feuilles isolées ayant absorbé de l'herbicide.

\section{MATÉRIEL ET MÉTHODES}

Les semences de la population résistante proviennent d'un champ de maïs en monoculture depuis 10 ans à Entraigues (Puy-de-Dôme) où, malgré un désherbage régulier à dose normale d'atrazine, l'effectif de la population a brusquement augmenté à partir de la septième annéc de traitement. En $1980,3500 \mathrm{~g} / \mathrm{ha}$ de matière active d'un mélange d'atrazine et de simazine ont été utilisés. Les semences de la population sensible proviennent d'un potager jamais désherbé près de Dijon (Côte-d'Or). Les individus utilisés ont été obtenus en serre, en jour long ( $16 \mathrm{~h}$ jour à $24{ }^{\circ} \mathrm{C} ; 8$ h nuit à $18^{\circ} \mathrm{C}$ ), après germination (à $20^{\circ} \mathrm{C}$ nuit, $30^{\circ} \mathrm{C}$ jour sous la même photopériode) des semences issues des parcelles d'origine.

Pour l'étude de la détoxication, 120 plantules de chaque population ont été placées dans du sable régulièrement submergé par une solution de HEwITT. Après $45 \mathrm{j}$, elles ont été prélevées avec précaution et placées dans des vases contenant de la solution nutritive aérée. Après $3 \mathrm{j}$, de l'atrazine technique dissoute dans un petit volume d'éthanol ( $4 \mathrm{ml} / 1$ de solution) est ajoutée à la solution pour obtenir une concentration finale de $20 \mathrm{mg}$ d'atrazine/l de solution. Après $24 \mathrm{~h}$ de traitement, les racines sont rincées et les plantes sont remises dans de la solution aérée normale. Quatre séries de prélèvements ont été réalisées : à la fin du traitement $\left(\mathrm{T}_{0}\right), 1 \mathrm{j}\left(\mathrm{T}_{1}\right), 2 \mathrm{j}\left(\mathrm{T}_{2}\right)$ et $6 \mathrm{j}$ après $\left(\mathrm{T}_{6}\right)$. Chaque prélèvement pour chaque population était composé de 3 répétitions de 10 individus. Les individus du dernier prélèvement n'avaient pas grandi d'une manière significative par rapport à la taille qu'ils avaient lors du traitement ; ceci évite le risque de sous-estimation de la concentration d'atrazine chez ces individus.

Pour mesurer le niveau de détoxication, nous avons, en fait, dosé l'évolution de la quantité d'atrazine absorbée qui est généralement bloquée dans les feuilles, car la disparition du produit dosable est directement corrélée à sa métabolisation. L'atrazine a été extraite selon une technique simplifiée de celle de Shimabukuro (1966). Les feuilles ont été broyées au broyeur Sorwall pendant $1 \mathrm{mn}$ environ dans du méthanol (10 volumes/poids des feuilles), puis filtrées sous vide. Après rinçage du matériel, l'extrait, additionné de $10 \mathrm{ml}$ d'eau, a été concentré jusqu'à $15 \mathrm{ml}$ environ et centrifugé $10 \mathrm{mn}$ à $10000 \mathrm{~g}$. Le surnageant a été extrait 2 fois par $10 \mathrm{ml}$ de chloroforme. Les fractions chloroformiques récupérées ont été évaporées à sec et le résidu repris dans $4 \mathrm{ml}$ de méthanol. Le dosage de l'atrazine (adapté de GAILlARDON, 1975) a été effectué par chromatographie en phase gazeuse avec une colonne Gas Chrom $Q$ DMCS $80 / 100$ de Carbowax $20 \mathrm{M}$ à 5 p. 100 , gaz vecteur $\mathrm{N}_{2}$ (pression d'entréc 1,6 bar), détecteur thermoionique, température du four constante $195{ }^{\circ} \mathrm{C}$, de l'injection et du détecteur, $250{ }^{\circ} \mathrm{C}$.

Les tests rapides de fluorescence ont été réalisés sur de jeunes feuilles selon un protocole que nous avons déjà décrit (DuCruet \& Gasquez, 1978). L'extraction des chloroplastes des 2 populations a été conduite selon la technique de NOBEL (1974), modifiée par ARNTZEN et al. (1974). L'activité traduite par le dégagement d'O $\mathrm{O}_{2}$ a été mesurée à l'électrode platine - argent, selon une technique précédemment utilisée (DUCRUET \& GASQUEZ, 1978).

\section{RÉSULTATS}

La figure 1 montre les valeurs des dosages d'atrazine dans chaque population. Les valeurs qui correspondent au dosage de produit au moment de l'arrêt du traitement $(0)$, sont comparables pour les 2 populations, ce qui indique un niveau d'absorption équivalent. Ces valeurs sont de l'ordre de celles rapportées pour différentes espèces de sétaires par THOMPSON (1972). $24 \mathrm{~h}$ après le traitement, la détoxication a déjà pratiquement atteint son maximum (fig. 1) et il ne reste environ que le quart de l'atrazine absorbée chez les 2 biotypes. Ceci est comparable aux valeurs obtenues sur des morceaux de feuilles, infiltrées d'atrazine marquée qui, après $8 \mathrm{~h}$, ont métabolisé $44 \mathrm{p} .100 \mathrm{du}$ produit (JENSEN et al., 1977). La dégradation est, par la suite, plus lente ; après $6 \mathrm{j}$, les valeurs sont comparables à celles du premier jour et pourraient correspondre au blocage de l'atrazine dans des sites inactifs.

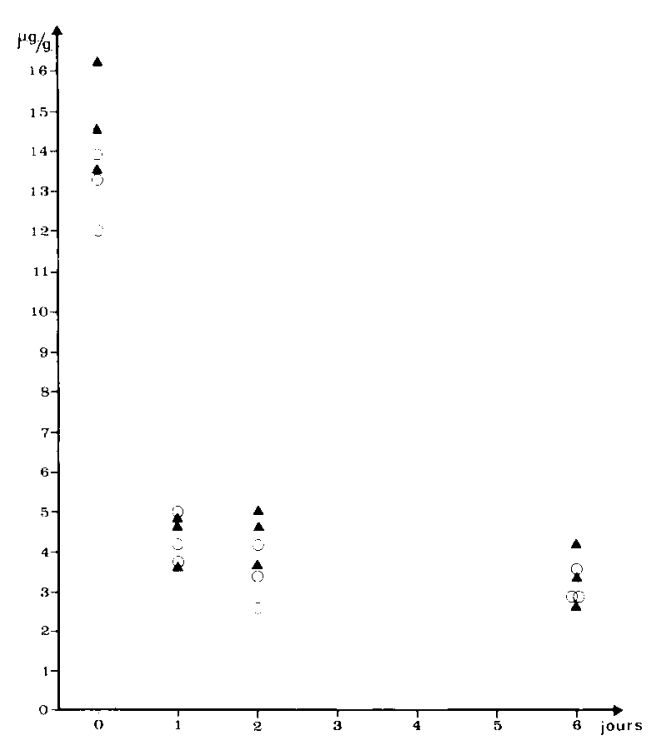

Figure 1

Variation des concentrations d'atrazine ( $\mu \mathrm{g} / \mathrm{g}$ de tissu frais) dans les feuilles en fonction du temps après un traitement de $24 \mathrm{~h}$. Résistant $\mathbf{\Delta}$ Sensible 0 .

Evolution of atrazine concentration ( $\mu \mathrm{g} / \mathrm{g}$ of fresh tissue) in leaves during several days after a 24 h treatment. $\Delta$ Resistant $O$ Susceptible.

Ainsi, tant pour l'absorption que pour la détoxication, les 2 populations ont un comportement très comparable. Il semble qu'il n'y ait pas de différence pour ce mode de résistance entre les individus résistants et ceux de la population jamais traitée, ce qui laisse penser que les traitements des années précédentes pourraient ne pas avoir sélectionné des individus à forte capacité de dégradation.

En revanche, l'observation de la fluorescence de feuilles isolées des individus résistants montre, après trempage dans l'atrazine, des courbes à bas niveau de fluorescence, caractéristiques des biotypes présentant des chloroplastes résistants (DuCRUET \& GASQueZ, 1978 ; GasQueZ \& BARRA- 
LIS, 1979 ; GASQUEZ \& COMPOINT, 1980). La fluorescence des feuilles d'individus sensibles est maximale, traduisant un blocage du transfert d'électrons (fig. 2).
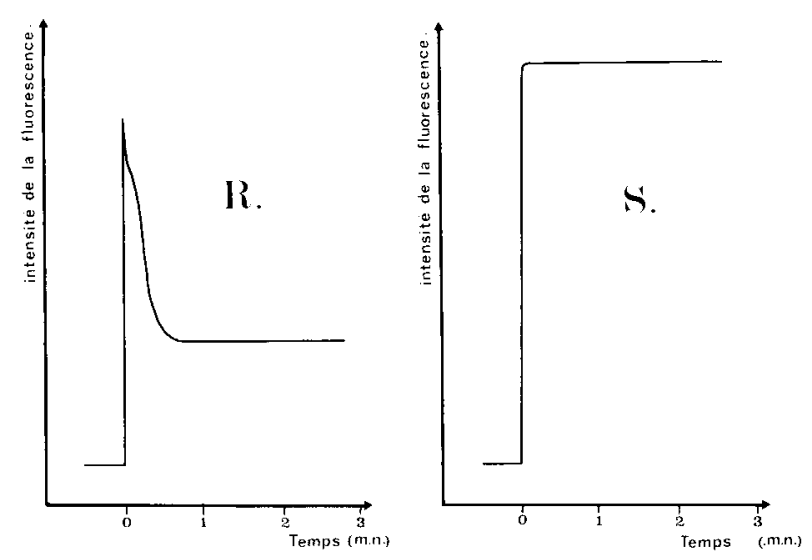

Figure 2

Intensité de la fluorescence de feuilles entières d'individus résistants $(R)$ et sensibles $(S)$ après $2 h$ de trempage dans de l'atrazine à 30 ppm. Le temps est compté à partir de l'ouverture du faisceau excitateur.

Fluorescence intensity of resistant $(R)$ and susceptible (S) leaves after two hours absorption of atrazine ( $(30 \mathrm{ppm})$. Time scale starts when light is given.

Les doses entraînant la demi-inhibition du dégagement d'oxygène de chloroplastes isolés de la population résistante (tabl. 1) sont du même ordre que les valeurs observées chez les individus d'espèces pour lesquels a été décrite la résistance chloroplastique. De même, les 2 biotypes sont sensibles au diuron dans les mêmes proportions (DUCRUET \& GASQUEZ, 1978). Les chloroplastes des sétaires résistantes sont donc environ 1000 fois plus résistants à l'atrazine que ceux de plantes sensibles. Ainsi, les résultats de fluorescence et de mesure de l'activité des chloroplastes montrent que les individus de la population résistante ont des chloroplastes insensibles à l'atrazine et que cette résistance est très certainement du même type que celle que l'on connaît chez les Dicotylédones.

\section{TABLEAU I}

Valeurs de demi-inhibition par l'atrazine (Molell) du dégagement d'oxygène de chloroplastes isolés - $R$ : résistants; $S$ : sensibles.

Concentration of atrazine and diuron required for 50 p. 100 inhibition of resistant $(R)$ and susceptible $(S)$ chloroplasts activity $(M / l)$

$\begin{array}{lcr} & \text { Atrazine } & \text { Diuron } \\ \mathrm{R} & 10^{-4} & 1,7 \times 10^{-7} \\ \mathrm{~S} & 1,5 \times 10^{-7} & 9 \times 10^{-8}\end{array}$

\section{CONCLUSION}

Malgré une grande résistance à l'atrazine de la population de Setaria viridis issue d'un champ de maïs, il n'existe entre les 2 populations étudiées aucune différence significative dans l'aptitude à métaboliser l'herbicide. La population sensible n'ayant jamais été traitée est certainement constituée d'un éventail de génotypes ayant des capacités à dégrader très variables, comme c'est le cas chez Echinochloa crus-galli (GASQUEZ \& COMPOINT, 1977).

Ainsi, les individus étudiés de la population résistante n'ont certainement pas été sélectionnés pour leur meilleure aptitude à détoxifier l'herbicide. En revanche, l'observation de la fluorescence de feuilles isolées et la mesure de l'activité de chloroplastes isolés, montrent que seuls les individus résistants présentent une résistance chloroplastique du même type que celle qui a été précédemment décrite chez des espèces incapables de dégrader. Ainsi, Setaria viridis est actuellement la seule espèce dont les individus d'une population réunissent les 2 types connus de résistance aux triazines. Cependant il se pourrait qu'il existe, chez d'autres espèces de Graminées, des populations qui aient à la fois des chloroplastes résistants et soient capables de dégrader plus ou moins l'herbicide. En effet, le comportement de ces populations qui doit être moins spectaculaire que chez les Dicotylédones (car au départ l'espèce est peu sensible), rend difficile la reconnaissance de la résistance chloroplastique. Ainsi, des biotypes d'autres espèces de Setaria ( $S$. glauca et $S$. faberii) que nous étudions actuellement semblent présenter ces caractéristiques.

Comme il a pu se développer un phénomène de résistance chez des espèces qui en étaient apparemment dépourvues $a$ priori, il pourrait, chez certaines espèces, se développer simultanément plusieurs types de résistance sur des individus différents. La prépondérance plus ou moins grande d'un type dépendra des fréquences relatives au départ des individus portant chaque caractère adaptatif et surtout des différences de valeur adaptative de chaque résistance. En revanche, il serait surprenant, à l'exception d'une mutation très récente, que les mêmes individus soient sélectionnés simultanément par 2 mécanismes très différents de résistance à un herbicide. Ainsi, le fait que les individus de Setaria viridis observés ne métabolisent pas plus que des sensibles n'ayant jamais été traités permet de penser que leur niveau de détoxication correspond au minimum de l'espèce et, qu'en fait, ils n'auraient été sélectionnés que par un seul mécanisme: la résistance chloroplastique. Ce qui permet de formuler l'hypothèse que nous cherchons à vérifier, selon laquelle il y aurait eu chez cette population, au cours des années successives de traitement, sélection de certains génotypes qui dégradent mieux sans qu'il y ait élimination complète d'autres génotypes parmi lesquels aura pu se développer la résistance chloroplastique. Ainsi, ce serait le phénomène rare, mais à très forte valeur adaptative, qui aurait pris, en moins de 10 ans, le pas sur le phénomène progressif et quantitatif apparemment avantagé a priori par le grand nombre d'individus qui le possèdent.

Reçu le $1^{\text {er }}$ juin 1981. Accepté le 26 août 1981.

\section{REMERCIEMENTS}

Nous tenons à remercier M. Rougeron (Limagrain) qui nous a gracieusement fourni les semences résistantes. 


\section{RÉFÉRENCES BIBLIOGRAPHIQUES}

Aigle N., Barralis G., Gasquez J., 1980. Quelques données sur la répartition de la résistance chloroplastique en France. VI ${ }^{i m e}$ Coll. Intern. Ecologie, Biologie et Systématique des Mauvaises Herbes, 367-377.

Arntzen C. J., Armond P. A., Zettinger C. S., Vernotte C., Briantais J. M., 1974. Lactoperoxydase - catalysed iodination of chloroplast membranes. Biochim. Biophys. Acta, 329, 339.

Ducruet J. M., Gasquez J., 1978. Observation de la fluorescence sur feuille entière et mise en évidence de la résistance chloroplastique à l'atrazine chez Chenopodium album L. et Poa annua L.. Chemosphere, 8, 691-696.

Gaillardon P., 1975. Etude des phénomènes de sorption entre deux triazines herbicides et des acides humiques. Weed Res., 15, 393-399.

Gasquez J., Barralis G., 1979. Mise en évidence de la résistance aux triazines chez Solanum nigrum L. et Polygonum lapathifolium L. par observation de la fluorescence de feuilles isolées. C.R. Acad. Sc. Paris D, 288, 1391-1393.

Gasquez J., Compoint J. P., 1977. Mise en évidence de la variabilité génétique infra-population par l'utilisation d'isoenzymes foliaires chez Echinochloa crus galli (L.) P.B.. Ann. Amélior. Plant., 27, (2), 267-278.

Gasquez J., Compoint J. P., 1980. Trois nouvelles mauvaises herbes résistant aux triazines en France: Amaranthus retroflexus L., Chenopodium polyspermum L., Polygonum persicaria L.. Chemosphere, 9, 39-43.
Jensen K. I. N., Stephenson G. R., Hunt L. A., 1977. Detoxification of atrazine in three Gramineae subfamilies. Weed Sci., 25, (3), 212-220.

Nobel P. S., 1974. Rapid isolation techniques for chloroplasts. In Colowick S. P. and Kaplan N. O.: Methods in Enzymology 31 Acad Press 600.

Ryan G. E., 1970. Resistance of common groundsel to simazine and atrazine. Weed Sci., 18, 614-616.

Shimabukuro R. H., 1968. Atrazine metabolism in resistant corn and sorghum. Plant Physiol., 43, 1925-1930.

Shimabukuro R. H., Kadunce R. E., Frear D. S., 1966. Dealkylation of atrazine in mature pea plants. J. agr. Food Chem., 14 (4), 392-395.

Souza Machado V., Arntzen C. J., Bandeen J. D., Stephenson G. R., 1978. Comparative triazine effects upon System II Photochemistry in chloroplasts of two common lambsquarter (Chenopodium album) biotypes. Weed Sci., $26: 318$.

Souza Machado V., Bandeen J. D., Stephenson G. R., Jensen K. I. N., 1977. Differential atrazine interference with the Hill reaction of isolated chloroplasts from Chenopodium album $\mathrm{L}$. biotypes. Weed Res., 17, 407-413.

Thompson Jr. L., 1972. Metabolism of chloro s-triazine herbicides by Panicum and Setaria. Weed Sci., 20 (6), 584-587. 